\title{
Automated generation of a four-dimensional model of the liver using warping and mutual information
}

\author{
K. M. Brock, ${ }^{\text {a) }}$ J. M. Balter, L. A. Dawson, and M. L. Kessler \\ Department of Radiation Oncology, University of Michigan Health Systems, Ann Arbor, Michigan 48109 \\ C. R. Meyer \\ Department of Radiology, University of Michigan Health Systems, Ann Arbor, Michigan 48109
}

(Received 2 October 2002; accepted for publication 2 April 2003; published 29 May 2003)

\begin{abstract}
The use of mutual information (MI) based alignment to map changes in liver shape and position from exhale to inhale was investigated. Inhale and exhale CT scans were obtained with intravenous contrast for six patients. MI based alignment using thin-plate spine (TPS) warping was performed between each inhale and exhale image set. An expert radiation oncologist identified corresponding vessel bifurcations on the exhale and inhale CT image and the transformation for identified points was determined. This transformation was then used to determine the accuracy of the MI based alignment. The reproducibility of the vessel bifurcation identification was measured through repeat blinded vessel bifurcation identification. Reproducibility [standard deviation (SD)] in the $L / R, A / P$, and $I / S$ directions was $0.11,0.09$, and $0.14 \mathrm{~cm}$, respectively. The average absolute difference between the transformation obtained using MI based alignment and the vessel bifurcation in the $L / R, A / P$, and $I / S$ directions was $0.13 \mathrm{~cm}(\mathrm{SD}=0.10 \mathrm{~cm}), 0.15 \mathrm{~cm}(\mathrm{SD}=0.12 \mathrm{~cm})$, and $0.15 \mathrm{~cm}(\mathrm{SD}-0.14 \mathrm{~cm})$, respectively. These values are comparable to the reproducibility of bifurcation identification, indicating that MI based alignment using TPS warping is accurate to within measurement error and is a reliable tool to aid in describing deformation that the liver undergoes from the exhale to inhale state. (C) 2003 American Association of Physicists in Medicine.
\end{abstract}

[DOI: $10.1118 / 1.1576781]$

Key words: deformation, mutual information, warping, liver radiotherapy

\section{INTRODUCTION}

The inclusion of organ deformation in treatment planning is of increasing interest as research begins to show the extent of the effects of physiological processes on the shape and position of organs. Standard treatment planning practice is to obtain one model of the patient (i.e., treatment planning CT scan) and assume that this shape and position represent the patient on every day of treatment.

In a recent study, we demonstrated that the liver shape changes during breathing. A four-dimensional model of the liver was developed based on multiple breath-held CT scans, and applying finite element modeling and finite element analysis $(\mathrm{FEA})^{1}$ Although this method was feasible, it was time consuming and user dependent.

Image alignment using mutual information (MI) as a metric has become a popular method to perform automatic alignment of two images since first being described by Viola and Wells in $1995 .^{2}$ Initial investigations used MI to align images from different modalities such as CT, MRI, and PET. ${ }^{3-5}$

Researchers then began to investigate the ability to align two image sets from the same patient to determine motion and changes in anatomic features. Kim et al. used MI to estimate setup error using a three-dimensional (3D) CT scan aligned to one or two two-dimensional radiographs. ${ }^{6}$ Carrillo et al. used a semiautomatic method to align MR images of the liver during thermal ablation of liver cancer. ${ }^{7}$ SanjayGopal et al. investigated the benefits of MI-based alignment to characterize the interval change in breast lesions on mammograms. ${ }^{8}$

Recently, incorporation of spline models into the MIbased alignment has permitted the inclusion of nonrigid body transformations. Studholme et al. estimated tissue deformation due to intracranial electrode implantation using B-spline MI-based alignment between successive functional MR images. ${ }^{9}$ Bruckner et al. investigated the advantages of using elastic matching for MI-based alignment of mammography. ${ }^{10}$ Meyer et al. has developed a method using thin-plate spline (TPS) MI-based alignment to quantify lesion changes between subsequent liver CT scans. ${ }^{11}$

The purpose of this study is to investigate the use of TPS MI-based deformable alignment for mapping changes in the shape and position of the liver between inhale and exhale breath held CT models of a patient.

\section{METHODS AND MATERIALS}

\section{A. Data collection}

Patient data were obtained under a clinical protocol approved by the University of Michigan Medical Center Internal Review Board. Two computed tomography (CT) scans were obtained for six patients. The first scan was obtained at end-exhale and the second at normal inhale. The patient's breath was held using an active breathing control device using a method previously described. ${ }^{12}$ All scans were acquired using a $3 \mathrm{~mm}$ aperture and a pitch of 2 , resulting in a CT 
resolution of $0.09 \mathrm{~cm}$ by $0.09 \mathrm{~cm}$ by $0.3 \mathrm{~cm}$. A helical CT scanner $(\mathrm{Ct} / \mathrm{i}$, General Electric, Milwaukee, WI) with a scan field of view of $48 \mathrm{~cm}$ was used for all scans. Scan C (barium sulfate) oral contrast and Omnipaque non-ionic IV contrast (Nycomed Inc.) was administered to the patients prior to their exhale scan. The inhale scan was taken immediately following the exhale scan, while some IV contrast still remained in the venous phase. The total scan time, for both scans, was approximately 3-5 min.

\section{B. Warping and mutual information method}

A mutual-information-based alignment software tool was developed by Meyer et al. MIAMI Fuse.@ ${ }^{11}$ This alignment is implemented using the Nelder-Mead simplex algorithm. ${ }^{13}$ The system was designed to manipulate reference points placed on two CT data sets, a reference data set and a homologous data set, to determine the required transformation to deform the homologous data set into the reference data set. Using this tool, Meyer et al. have shown that 24 control points are sufficient to map $2 \mathrm{CT}$ scans acquired from the same patient at different times. Implanted markers were used to determine the accuracy of the alignment. Despite one of the CT scans having a slice thickness of $1 \mathrm{~cm}$ and yielding the largest error, the rms for 11 coils in 4 patients was 3.0 $\mathrm{mm} .{ }^{14}$ This algorithm and method are used here to align an inhale CT scan to an exhale CT from the same patient to determine the deformation the liver undergoes during normal breathing.

Full attention of the registration was concentrated on the liver by cropping out unwanted data. A probabilistic liver model, created from 32 patients, most with liver disease, was used to automatically segment the liver from the surrounding anatomy. ${ }^{15}$ Figure 1 shows an example of a CT slice prior to automatic segmentation of the liver and after automatic segmentation of the liver. The probabilistic liver model, with a threshold set at $20 \%$, was mapped onto the reference data set (the exhale CT) to remove most nonliver data. The probabilistic atlas is also used to automatically place 24 control points onto the reference image. Figure 2 shows the projection of the 24 points onto a model of one patient's liver. Clearly selection of control point loci in the atlas affects registration. Generally, the control points were selected to support control of both internal hepatic structures as well as boundaries. Control point density is approximately uniformly distributed throughout the liver. Local control in any region can be increased by increasing the control point density within the same region. Nevertheless, the fact that the liver, kidneys, and spinal cord of 31 patients could be mapped into the same reference with significantly lower variance than that provided by the full affine mapping, suggests that the actual location of any one control point is not so significant. The user then manually places four control points onto the homologous data set (the inhale CT) in roughly the same place as the first four control points in the reference image. These 4 points are used to identify the orientation of the patient during the two scans, i.e., prone/supine, head/feet-first. Given that the resulting initial registration is within the broad

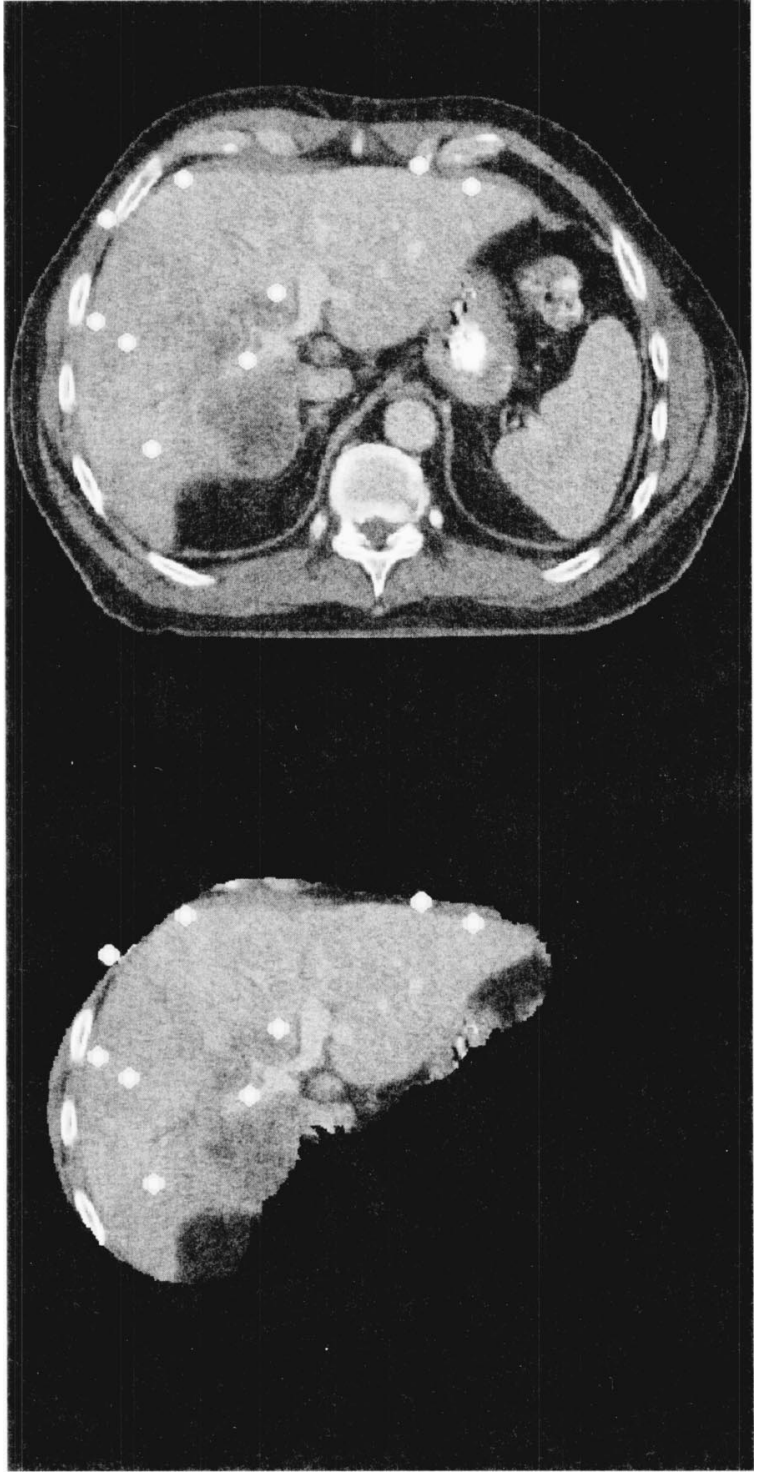

FIG. 1. CT slice of the liver before and after automatic segmentation with control points (shown in light gray).

capture range of MIAMI Fuse, the accuracy of the initial placement of the 4 points onto the inhale scan does not effect the accuracy of the final outcome of the MI-based alignment.

First the algorithm iteratively computes the optimal full affine transform between the two data sets using only the first four control points and a decimated version (every third voxel in $x$ and $y$, and every other plane in $z$ ) of the reference. The optimization is repeated until three solutions have the same MI within 0.01 bit. Then the algorithm maps the first 12 control points onto the homologous data set using the previously MI optimized registration and a hierarchical addition of the number of degrees of freedom in a multiresolution approach using every other voxel in $x$ and $y$, and every plane in $z$ at this state. Using the Nelder-Mead simplex algorithm, the $\mathrm{N} \times 3$ parameter space ( $n$ control points, $x, y, z$, coordinates) is searched. For each sampled configuration, a thin-plate spline-based warp is applied to the segmented exhale CT data at the tested resolution. From this deformation 


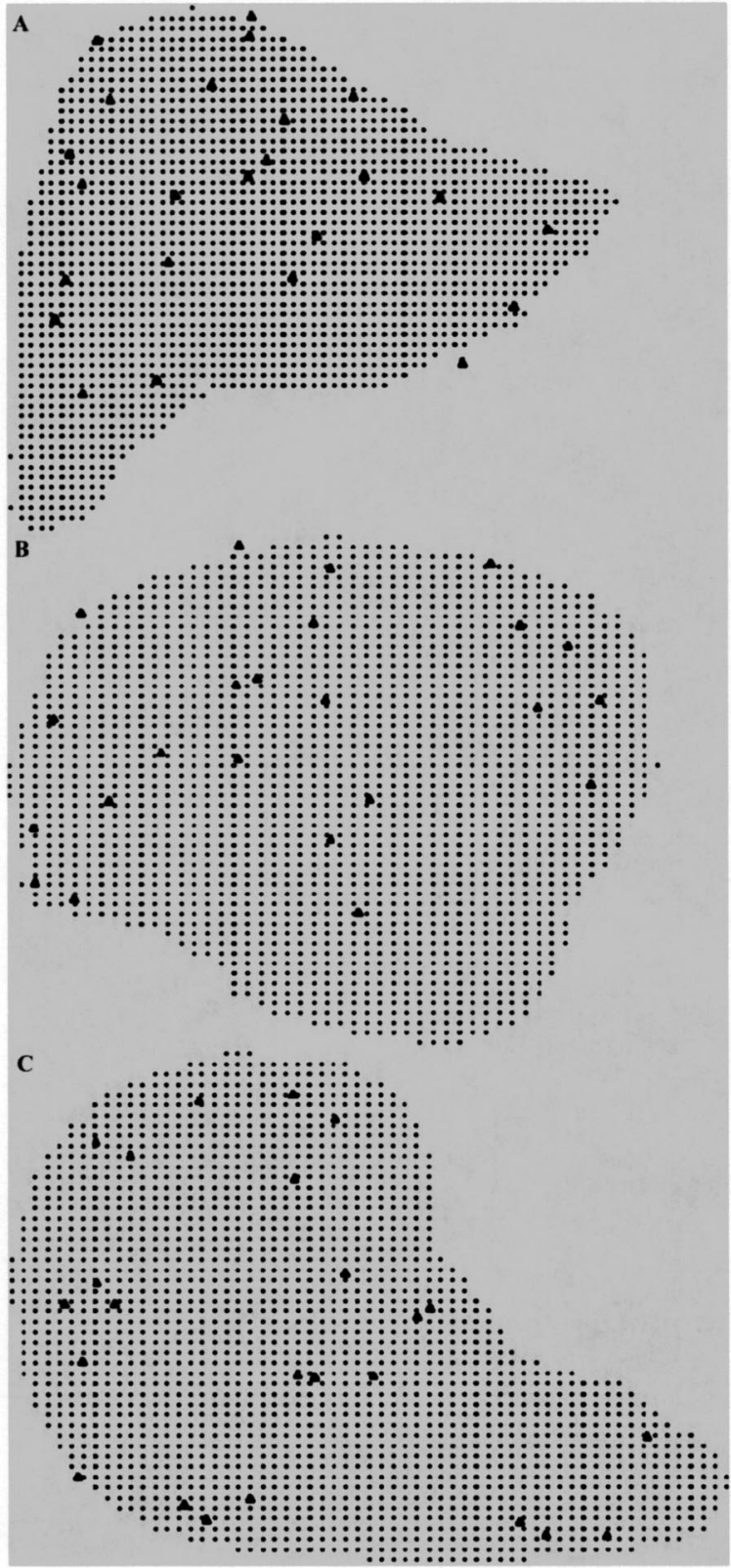

FIG. 2. The location of the 24 control points in the (A) coronal, (B) sagittal, and (C) axial plane for one patient. $(\mathbf{O})=$ Liver, $\triangle=24$ points).

intensity distribution, the joint intensity distribution with the inhale model is generated and the mutual information is computed. The configuration of control points that maximizes the mutual information to within 0.001 bit is selected as optimal. Finally the algorithm then includes all 24 points at the same decimation as used previously for 12 control points and iterates until three solutions have the same MI within 0.0001 bit. The multiresolution registration decreases the amount of time required for the registration while maintaining the accuracy specified in the last stage resolution. The algorithm typically requires 18700 iterations and takes approximately $5.5 \mathrm{~h}$ of clock time on a DS20 (DEC Alpha SERVER running DEC Unix).

Once the MI-based alignment of the two CT scans is completed, the transformation of the 24 control points can be

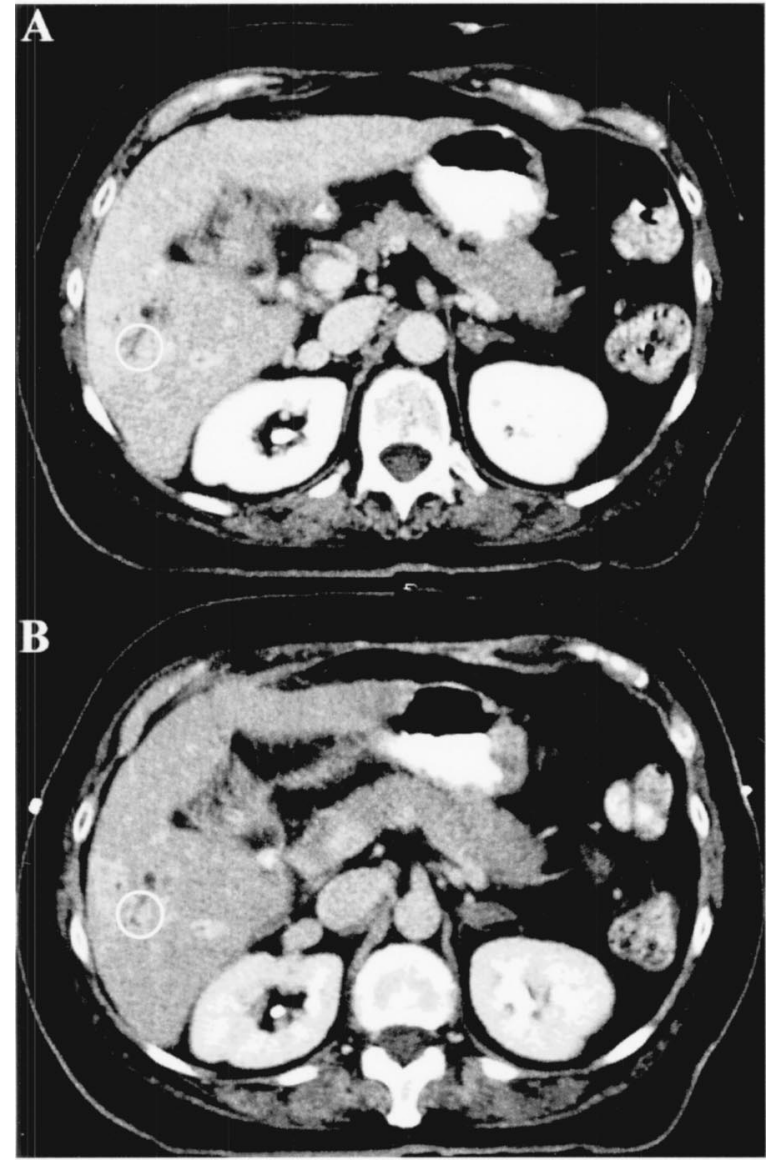

FIG. 3. Example bifurcation on exhale (A) and inhale (B) CT slice.

used to find the transformed location of any 3D coordinate as well as the bending energy for the entire liver. The bending energy is a representation of the amount of deformation that occurs between exhale and inhale states. ${ }^{16}$

\section{Evaluation of accuracy}

Given that both the exhale and inhale CT scans had contrast in the venous stage, vessel bifurcations were visible on both data sets. An expert radiation oncology physician (LAD) identified vessel bifurcations on the exhale image and their corresponding location on the inhale image. Occasionally, bile duct bifurcations were identified in the same manner. The coordinates of the bifurcations were obtained from both images and the displacement was calculated. The deformation map was then used to determine the MI displacement for the location of the bifurcation on the exhale image. The displacements for each bifurcation based on the CT scans and MI were then compared to determine the accuracy of the MI registration. An example of a bifurcation on the exhale and inhale CT image is shown in Fig. 3.

The reproducibility of the identification of the bifurcations was also measured. The physician was shown the previous placement of the bifurcation on the exhale image and instructed to identify the corresponding bifurcation on the inhale image. The three dimensional coordinates were obtained using UMPLAN. This procedure was repeated six times 


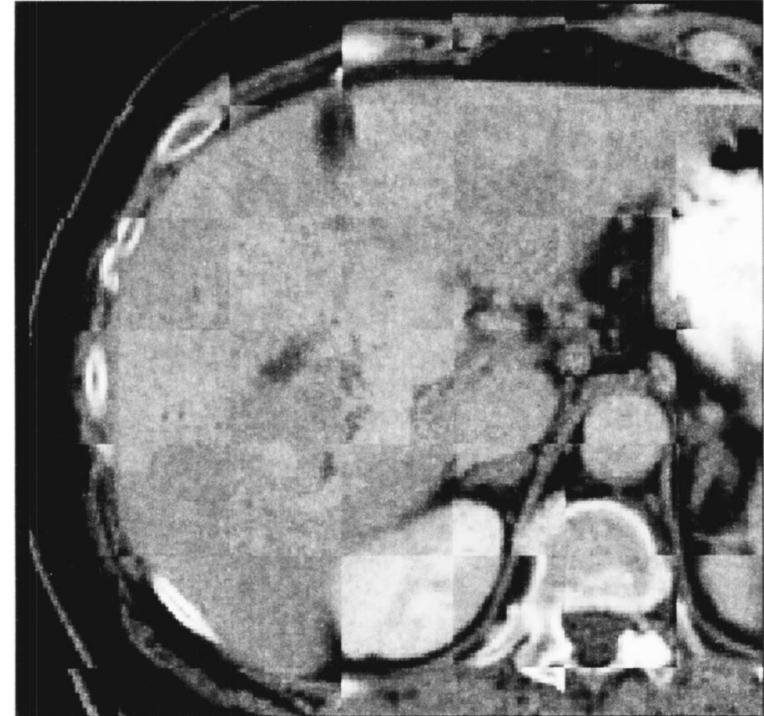

FIG. 4. Checkerboard comparison between exhale CT slice and MI-aligned inhale CT slice.

for 30 markers on three patients, randomly selected. Generally, there was a gap of a minimum of seven days between reproducibility measurements. The physician was blinded to the patient data set and the results from the MI transformation. The difference between repeated bifurcation coordinates was then calculated, and reproducibility was described using descriptive statistics.

\section{Liver model}

A geometric model of the liver was created using a volume region of interest (VROI) data representation generated in UMPLAN, the in-house treatment planning system at the University of Michigan Medical Center, Department of Radiation Oncology. This is a voxel based data set representing only voxels that contain the anatomy contoured as liver. Each voxel is $0.316 \mathrm{~cm}$ by $0.316 \mathrm{~cm}$ by $0.316 \mathrm{~cm}$ in size. The coordinate of the center of each voxel is obtained and the displacement is determined from the deformation map described previously. This sampling of the liver is only used to determine the transformation of points in the liver after alignment using the MI-based algorithm. This sampling is not used for the MI-based alignment or the identification of the liver vessel bifurcations.

\section{RESULTS}

MI-based alignment between the exhale and inhale scan was performed on six CT data sets. Vessel bifurcations were also identified and marked by the expert physician on all exhale and inhale images.

The results from the MI-based alignment were verified visually using a checkerboard comparison of the exhale CT scan and the aligned inhale CT scan as shown in Fig. 4. Figure 3 shows excellent agreement between the surface of the liver and the internal features visible from the contrast
TABLE I. Difference between displacement of points obtained using MIbased alignment and vessel bifurcation locations on CT scans. Absolute difference, mean $(\mathrm{SD})(\mathrm{cm})$

\begin{tabular}{ccccc}
\hline \hline Case No. & No. VB & $L / R$ & $A / P$ & $I / S$ \\
\hline 1 & 12 & $0.16(0.10)$ & $0.13(0.12)$ & $0.19(0.11)$ \\
2 & 14 & $0.08(0.07)$ & $0.18(0.14)$ & $0.08(0.05)$ \\
3 & 18 & $0.13(0.10)$ & $0.13(0.11)$ & $0.11(0.08)$ \\
4 & 10 & $0.12(0.07)$ & $0.11(0.08)$ & $0.31(0.26)$ \\
5 & 8 & $0.15(0.09)$ & $0.18(0.15)$ & $0.12(0.09)$ \\
6 & 6 & $0.22(0.12)$ & $0.18(0.14)$ & $0.11(0.09)$ \\
Avg. & & $0.13(0.10)$ & $0.15(0.12)$ & $0.15(0.14)$ \\
\hline \hline
\end{tabular}

agent. The external surface of the patient as well as other anatomy in the slice are not aligned, as these regions are not included in the alignment process.

The displacement of the liver between exhale and inhale was determined for the center of each voxel from the geometric model obtained from the VROI file with a voxel size of 0.316 by 0.316 by $0.316 \mathrm{~cm}$. The range of displacement and average over all the patients in the $L / R, A / P$, and $I / S$ directions were -1.41 to $2.65 \mathrm{~cm}$. (Avg: $0.018 \mathrm{~cm}$ ), -2.87 to $-0.62 \mathrm{~cm}$, (Avg: $-0.38 \mathrm{~cm}$ ), and -3.13 to $0.45 \mathrm{~cm}$, (Avg: $-0.85 \mathrm{~cm}$ ), respectively. The bending energy ranged from 10.8 to 30.2 , with an average of 18.6. The bending energy that results from a 10 by 10 by $10 \mathrm{~cm}$ cube with one corner point displaced by $1,2.5$, and $5 \mathrm{~cm}$ is $0.8,5.0$, and 20.1 , respectively.

The displacement for each identified vessel bifurcation was determined via CT scan by calculating the difference between the location on the exhale scan and the inhale scan. The displacement of each bifurcation was also determined via MI-based alignment. The average difference in the $L / R, A / P$, and $I / S$ directions between the two methods of calculating the displacement is shown in Table I. The number of vessel bifurcations identified is also shown for each case. The number of vessel bifurcation identified ranged from 6 to 18. The average difference in the $L / R, A / P$, and $I / S$ directions ranged from 0.08 to $0.22 \mathrm{~cm}, 0.11$ to $0.18 \mathrm{~cm}$, and 0.08 to $0.31 \mathrm{~cm}$, respectively. The average difference over all the patients in the $L / R, A / R$, and $I / S$ direction was $0.13 \mathrm{~cm}$ $(\mathrm{SD}=0.10 \mathrm{~cm}), 0.15 \mathrm{~cm}(\mathrm{SD}=0.12 \mathrm{~cm})$, and $0.15 \mathrm{~cm}(\mathrm{SD}$ $=0.14 \mathrm{~cm}$ ).

The reproducibility vessel bifurcation identification was determined for ten randomly selected bifurcations for the first three patients studied. The test was repeated six times in 1 week intervals. The reproducibility over the three patients in the $L / R, A / P$, and $I / S$ directions are $0.11,0.09$, and 0.14 $\mathrm{cm}$, respectively.

A box plot graphing the accuracy of the MI-based alignment and the accuracy of selecting the vessel bifurcations is shown in Fig. 5.

A paired t-test was performed to determine if there was a significant difference between the displacement, for each marker, obtained using MI-based alignment and using vessel bifurcation location on inhale and exhale $\mathrm{CT}$ scans and the reproducibility of the selection of the vessel bifurcation. A significant difference between the two is indicated by a $p$ 


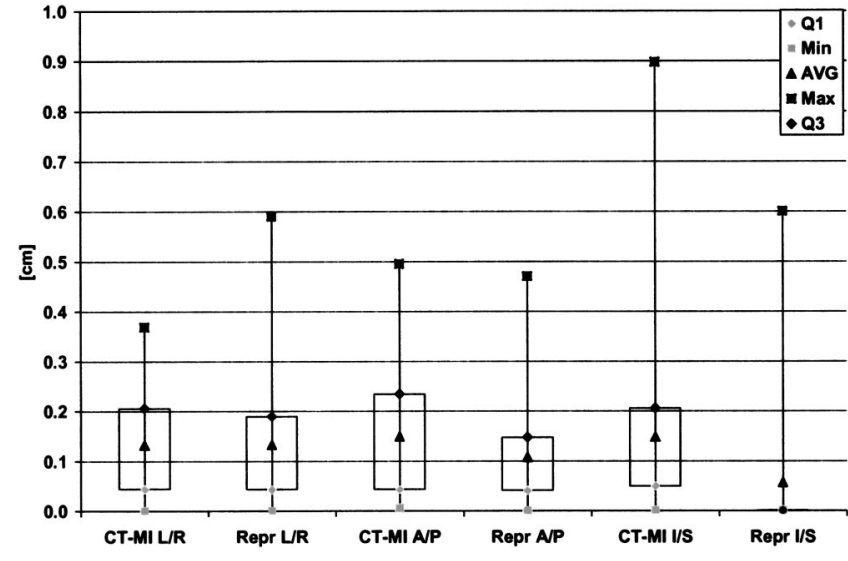

Fig. 5. Box plot representing the absolute difference between the displacement calculated using MI and vessel bifurcations (CT-MI) for each direction $(L / R, A / P$, and $I / S)$ and the accuracy of selecting the bifurcation (Repr) for each direction $(L / R, A / P$, and $I / S)$.

value less than 0.05 . The $p$ value was greater than 0.322 in all three directions. Thus, the results are not significantly different.

\section{DISCUSSION}

MI-based alignment using affine transformation and TPS warping is an attractive replacement for manual alignment aided by FEA for the creation of a deformation map of the liver. TPS-based deformations are very similar to the linear elastic model used in the FEA method. The use of TPS further removes the dependence of the result on assumed material properties. The process is completely automated with the exception of the placement of four orientation markers and the analysis is completely user independent. The automation and user independence make it ideal for clinical implementation.

MI-based alignment has been shown to be sufficiently accurate based on the comparison to change in vessel bifurcation position between exhale and inhale. The average difference between the displacement determined using MI-based alignment and the displacement calculated using the selection of corresponding vessel bifurcations on each data set is less than or equal to $0.31 \mathrm{~cm}$ in all three directions for each individual patient. The average over all the patients was less than or equal to $0.15 \mathrm{~cm}$ in all directions. The average difference between the alignment from MI and the vessel bifurcations (range: $0.13-0.15 \mathrm{~cm}$ ) is on the same order as the reproducibility of the location of the vessel bifurcation (range: $0.09-0.14 \mathrm{~cm}$ ). A paired t-test indicates that the difference between the displacement using MI or vessel bifurcations is not significant compared to the uncertainty in locating the vessel bifurcations. The accuracy of the MI-based alignment and the reproducibility of selecting the vessel bifurcations is less than the magnitude of average motion of the liver in the $A / P$ and $I / S$ directions, -0.38 and -0.85 $\mathrm{cm}$, respectively. It is possible, however, that the motion in the $L / R$ direction is not substantial, as the average motion in this direction, $-0.018 \mathrm{~cm}$, is less than the accuracy of the
MI-based alignment, $0.10 \mathrm{~cm}$. This indicates that the TPS is a sufficient surrogate for the appropriate liver material properties and that the method creates an accurate deformation map of the liver as it changes position and shape due to breathing.

One limitation of this study is the accuracy of the vessel bifurcation identification for evaluation of accuracy of alignment. There is an inevitable time delay between the acquisition of the two data sets (generally $<30 \mathrm{~s}$ ), so the contrast is at a different point of the venous phase on each scan. We have reported the reproducibility bifurcation identification. However, we are unable to determine if there is a systematic error in the selection of the same bifurcation point on each data set. We believe any potential systematic error is small, but this can only be confirmed through analysis using implanted fiducial markers. The accuracy of MI to align the liver from inhale to exhale, as measured using the vessel bifurcation position change is on the same order as the accuracy reported by Meyer et al., rms error's linear regression line of $3.0 \mathrm{~mm}$, using 11 implanted coils in four patients, suggesting that any systematic error in bifurcation identification is small.

One application for the method described to create a deformation map using MI-based alignment is to incorporate deformation into dose calculations. The MI-based alignment results do not describe intermediate positions of the liver, therefore defaulting to the use of a model to estimate these states. Research by Seppenwoolde et al. has shown that hysteresis occurred during breathing in $50 \%$ of the lung patients analyzed. ${ }^{17}$ One can speculate that this type of nonlinear behavior has the potential to affect the liver as well. Further investigation is necessary to investigate the importance of including such effects in the liver.

\section{ACKNOWLEDGMENTS}

This work was supported by NIH P01-CA87634, NIH P01-CA59827, and a grant from the Walther foundation.

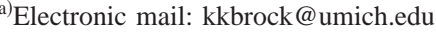

${ }^{1}$ K. K. Brock et al., "Technical note: Creating a four-dimensional model of the liver using finite element analysis," Med. Phys. 29, 1403-1405 (2002).

${ }^{2}$ P. A. Viola and W. M. Wells, "Alignment by maximization of mutual information," in Proceedings of the Fifth International Conference on Computer Vision, IEEE95CH35744, 16-23 (1995).

${ }^{3}$ W. M. Wells, 3rd et al., "Multi-modal volume registration by maximization of mutual information," Med. Image Anal 1, 35-51 (1996).

${ }^{4}$ C. Studholme, D. L. Hill, and D. J. Hawkes, "Automated threedimensional registration of magnetic resonance and positron emission tomography brain images by multiresolution optimization of voxel similarity measures," Med. Phys. 24, 23-35 (1997).

${ }^{5} \mathrm{~F}$. Maes et al., "Multimodality image registration by maximization of mutual information," IEEE Trans. Med. Imaging 16, 187-198 (1997).

${ }^{6} \mathrm{~J}$. Kim et al., "A feasibility study of mutual information based setup error estimation for radiotherapy,” Med. Phys. 28, 2507-2517 (2001).

${ }^{7}$ A. Carrillo et al., "Semiautomatic 3-D image registration as applied to interventional MRI liver cancer treatment," IEEE Trans. Med. Imaging 19, 175-185 (2000).

${ }^{8}$ S. Sanjay-Gopal et al., "A regional registration technique for automated interval change analysis of breast lesions on mammograms," Med. Phys. 26, 2669-2679 (1999).
} 
${ }^{9} \mathrm{C}$. Studholme et al., "Estimating tissue deformation between functional images induced by intracranial electrode implantation using anatomical MRI," Neuroimage 13, 561-576 (2001).

${ }^{10}$ T. Bruckner, R. Lucht, and G. Brix, "Comparison of rigid and elastic matching of dynamic magnetic resonance mammographic images by mutual information" Med. Phys. 27, 2456-2461 (2000).

${ }^{11} \mathrm{C}$. R. Meyer et al., "Demonstration of accuracy and clinical versatility of mutual information for automatic multimodality image fusion using affine and thin-plate spline warped geometric deformations," Med. Image Anal 1, 195-206 (1997).

${ }^{12}$ K. K. Brock et al., "Inclusion of organ deformation in dose calculations," Med. Phys. 30, 290-295 (2003).

${ }^{13}$ W. H. Press et al., Numerical Recipes in C: The Art of Scientific Computing (Cambridge University Press, Cambridge, 1988).
${ }^{14}$ C. R. Meyer et al., "Method for quantifying volumetric lesion change of interval liver CT examinations," IEEE Trans. Med. Imaging (in press).

${ }^{15}$ H. Park, P. H. Bland, and C. R. Meyer, "Construction of an abdominal probabilistic atlas and its application in segmentation," IEEE Trans. Med. Imaging (in press).

${ }^{16}$ F. L. Bookstein, "Principal warps: Thin-plate splines and the decomposition of deformations," IEEE Trans. Pattern Anal. Mach. Intell. 11, 567-585 (1989).

${ }^{17}$ Y. Seppenwoolde et al., "Precise and real-time measurement of 3D tumor motion in lung due to breathing and heartbeat, measured during radiotherapy," Int. J. Radiat. Oncol., Biol., Phys. 53, 822-834 (2002). 